\title{
A Pendulum-like Low Frequency Electromagnetic Vibration Energy Harvester Based on Polymer Spring and Coils
}

\author{
Yunjia Li ${ }^{1, *(\mathbb{D})}$, Xinyi Wang ${ }^{1}{ }^{\mathbb{D}}$, Shuhan Zhang ${ }^{1}$, Chenyuan Zhou ${ }^{1} \mathbb{D}$, Dayong Qiao $^{2}$ and Kai Tao ${ }^{2, *} \mathbb{C}$ \\ 1 School of Electrical Engineering, Xi'an Jiaotong University, Xi'an 710049, China; \\ xywang@stu.xjtu.edu.cn (X.W.); zhangshuhan123@foxmail.com (S.Z.); zhouchenyuan@stu.xjtu.edu.cn (C.Z.) \\ 2 Micro and Nano Electromechanical Systems Laboratory, Northwestern Polytechnical University, \\ Xi'an 710072, China; dyqiao@nwpu.edu.cn \\ * Correspondence: liyunjia@xjtu.edu.cn (Y.L.); taokai@nwpu.edu.cn (K.T.)
}

Citation: Li, Y.; Wang, X.; Zhang, S.; Zhou, C.; Qiao, D.; Tao, K.

A Pendulum-like Low Frequency Electromagnetic Vibration Energy Harvester Based on Polymer Spring and Coils. Polymers 2021, 13, 3380. https://doi.org/10.3390/ polym13193380

Academic Editor: Anowarul Habib

Received: 15 September 2021 Accepted: 24 September 2021 Published: 30 September 2021

Publisher's Note: MDPI stays neutral with regard to jurisdictional claims in published maps and institutional affiliations.

Copyright: (c) 2021 by the authors. Licensee MDPI, Basel, Switzerland. This article is an open access article distributed under the terms and conditions of the Creative Commons Attribution (CC BY) license (https:/ / creativecommons.org/licenses/by/ $4.0 /)$.

\begin{abstract}
This paper presents a low-frequency electromagnetic vibrational energy harvester (EVEH) with two degrees of freedom and two resonant modes. The proposed EVEH is based on a disc magnet suspended in a pendulum fashion by a polymeric spring between two sets of polymer coil stacks. The fabricated EVEH is capable of harvesting vibration energy on two directions with an extended bandwidth. With a sinusoidal acceleration of $\pm 1 \mathrm{~g}$ on $\mathrm{Z}$ direction, a peak-to-peak closed-circuit output voltage of $0.51 \mathrm{~V}$ (open-circuit voltage: $1 \mathrm{~V}$ ), and an output power of $35.1 \mu \mathrm{W}$ are achieved at the resonant frequency of $16 \mathrm{~Hz}$. With a sinusoidal acceleration of $\pm 1.5 \mathrm{~g}$ on $\mathrm{X}$ direction, a peakto-peak output voltage of $0.14 \mathrm{~V}$ and power of $2.56 \mu \mathrm{W}$ are achieved, at the resonant frequency of $20 \mathrm{~Hz}$.
\end{abstract}

Keywords: polymer beams; vibration sensors; vibration energy harvester

\section{Introduction}

Electromagnetic vibrational energy harvesters (EVEHs) are devices that convert ambient vibrations to electrical power, often by the relative movement between a permanent magnet and a solenoid coil [1-4]. Compared with its piezoelectric and electrostatic counterparts, the EVEHs possess advantages such as simpler design and structure, higher output power, and easier fabrication [5]. The EVEHs can be implemented by using macroscopic components, printed circuit board (PCB) technology [6-9], and microelectromechanical systems (MEMS) technologies [10-12].

The primary goals for designing the EVEHs are high output power, small volume, and low cost. However, in order to be used in practical applications, it is essential to match the resonant frequency of the EVEHs to the ambient vibration. It is known that the ambient vibrations where EVEHs can be used are mostly located in the frequency range of $1-200 \mathrm{~Hz}$ [11-14]. Therefore, it is desirable to implement EVEHs with low resonant frequencies. However, the stiffness of springs scales inversely with the geometrical dimensions, hence it is often difficult to reduce the resonant frequency (in direct proportional to the square root of the spring stiffness) of the miniaturized EVEHs. The conventional strategies to reduce the resonant frequency is to use low-stiffness material as springs or use folded spring design [11,14], which usually results in EVEHs with resonant frequency above $100 \mathrm{~Hz}$. EVEHs with resonant frequency lower than $100 \mathrm{~Hz}$ is less reported [15].

Besides the low frequency nature of ambient vibrations, many of them are multidirectional, such as the movement of human, vehicles (ships and airplanes), and cables. In addition, many of the vibrations vibrates on the horizontal direction with respect to the ground, such as power transformers. However, most of the reported EVEHs are based on unidirectional operation solely on the vertical direction. Therefore, it is desirable to obtain EVEHs that can harvest energy from vibrations of different directions, especially on the horizontal direction. Nevertheless, energy harvesters capable of harvesting vibrations on 
different directions are not so often reported. The reported multi-directional devices are mainly piezoelectric energy harvesters based on a cantilever structure [16-18]. EVEHs for multi-directional vibrations have rarely been reported, according to the best knowledge of the authors.

It is intuitive and straightforward to design and implement a multi-directional VEH by using a cantilever structure because its free end can move in any direction. Nevertheless, piezoelectric cantilevers cannot be implemented with large proof mass in order to avoid cracks and initial deflection, which limits the lower bound of its resonant frequency. In this work, we adopt the single-end structural feature of the cantilevers, but change the operation direction: suspending a permanent magnet in a pendulum-like fashion by a single flexible polymeric spring between two sets of stacked polymer coils. Compared to traditional EVEHs based on cantilever structures, the polymer single-string spring structure enables the integration of large proof mass, low operation frequency, and robustness of the device without the concern of crack or initial deflection. The stacked polymer coils enable integration of high-density coils within a small distance in the vicinity of the magnet, inducing large output voltage at low acceleration levels. The proposed EVEH is capable of generating rectifiable output voltage at very low frequency on two directions with two resonant modes, which provides wider applicability. Moreover, the proposed structure is shown to operate reliably at high acceleration levels. The design, fabrication, and characterization of the EVEH device will be presented and discussed.

\section{System Design}

\subsection{Concept of the EVEH}

The combined usage of the pendulum structure, the polymer-based springs and stacked coils enables excellent performance and robustness of the proposed EVEH. A schematic illustration of the proposed EVEH device is shown in Figure 1. It is composed of disc permanent magnets suspended like a pendulum by a soft polyimide spring, between two sets of high-density polymer coil stacks. The magnet suspended between two coil stacks is composed of two identical disc N52 NdFeB magnets bound to each other by magnetic force. A thin circular polyimide layer connected to the spring is sandwiched between the two magnets. The spring is anchored to the polyimide frame clamped between two rigid FR4 frames. Each of the polymer coil stacks consists of 14 identical polymer planar coil layers connected in series, forming a high-density 3D coil stack [7]. Fourteen is the maximum number of layers that can be included in the device without open-circuit fault. The advantage of the coil stacking technology is low cost, batch fabrication, and easy assembly. The magnet layer and the coil stacks are assembled by using bolts and spacers. When the EVEH is subjected to external accelerations, the magnet oscillates and generates a changing magnetic field in the coils, which induces voltage in the coils. When the acceleration on $X$ direction is applied, the magnet oscillates in a torsional mode (torsion of the spring). When the acceleration on $\mathrm{Z}$ direction is applied, the magnet oscillates in a bending mode (bending of the spring). The proposed EVEH is not sensitive to the vibration on $Y$ direction, because the $Y$-direction movement of the magnet involves axial elongation of the spring, which requires a large load to take place.

The polyimide spring has very low stiffness compared to the cantilever-based energy harvesters. However, due to the flexible nature of polyimide, the spring is rather robust compared to many of the cantilever structures made of brittle materials (e.g., silicon and piezoelectric ceramics). Therefore, the polyimide spring is capable of supporting large movement of the magnet. When excited by Z-direction vibration, the magnet will bend the spring and oscillate in $Z$ direction. At high acceleration levels, collisions between the magnet and the coil stack may occur in each oscillation cycle. This is different from most of the oscillating structures used for energy harvesting. The collisions between the magnet and the coil stack can be avoided by increasing the coil-magnet distance. However, the energy harvesting efficiency of the EVEH is significantly reduced when the coil-magnet distance increases, as a result of the exponentially decaying magnetic field outside the 
permanent magnet. In one of our previous study on the effect of the coil-magnet distance, the output voltage of reduces from $1.57 \mathrm{~V}$ at the coil-magnet distance of $0.71 \mathrm{~mm}$ [7] to around $0.5 \mathrm{~V}$ at $4 \mathrm{~mm}$. This is a deteriorative effect especially for the EVEH working under low acceleration levels, which is the case for most of the ambient vibrations. Therefore, the designed coil-magnet distance is $2.5 \mathrm{~mm}$, which is an intermediate value between $0.7 \mathrm{~mm}$ that we often use and $4 \mathrm{~mm}$ which is almost the lowest limit for the coil to harvest energy from the magnet. The value of $2.5 \mathrm{~mm}$ maintains a medium energy harvesting efficiency at a moderate collision level. The collision between the magnet and the coil may bring reliability issues after a long period of operation, and will be investigated in the future.

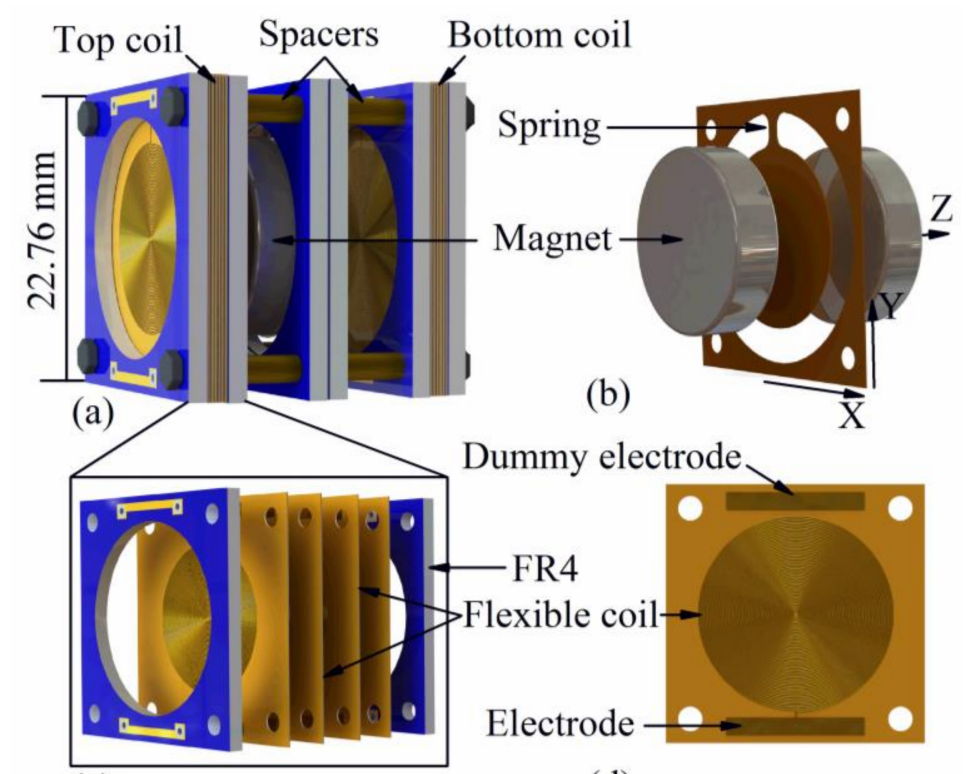

(c)

(d)

Figure 1. Schematic illustration of the: (a) EVEH device; (b) pendulum-like suspended magnet by polymer spring; (c) stacked polymer coils; and (d) a layer of polymer coils.

\subsection{System-Level Model}

Under the assumptions that the spring material is linearly elastic and that the shear deformation and rotary inertia are negligible, the differential equation of motion for a spring under axial load is given by [19]:

$$
E I \frac{\partial^{4} y}{\partial x^{4}}+F \frac{\partial^{2} y}{\partial x^{2}}+\rho A \frac{\partial^{2} y}{\partial t^{2}}=0
$$

where $y=y(x, t)$ is the transverse displacement of the spring located at the distance $\mathrm{x}$ from the left-hand end, $F$ is the axial load. $E I$ is the flexural rigidity of spring, $A$ is the cross-sectional area, and $\rho$ is the mass density of the spring material. If $F=0$, Equation (1) reduces to the classical solution.

When the spring performs harmonic oscillations $y(x, t)=Y(x) \cdot \cos (\omega t)$ the above expression becomes:

$$
E I \frac{\mathrm{d}^{4} Y(x)}{\mathrm{d} x^{4}}+F \frac{\mathrm{d}^{2} Y(x)}{\mathrm{d} x^{2}}-\rho A \omega^{2} Y(x)=0
$$

where $\omega$ is the circular natural frequency. By introducing the dimensionless spring coordinate $\bar{x}=x / l$, the solution of Equation (2) is:

$$
\bar{Y}(\bar{x})=A \cosh \left(\alpha_{1} \bar{x}\right)+B \sinh \left(\alpha_{1} \bar{x}\right)+C \cos \left(\alpha_{2} \bar{x}\right)+D \sin \left(\alpha_{2} \bar{x}\right)
$$


where $A, B, C$, and $D$ are constant coefficients that can be obtained from certain boundary conditions, and $\alpha_{1}$ and $\alpha_{2}$ are defined as:

$$
\begin{aligned}
& \alpha_{1}=l \sqrt{-\frac{F}{2 E I}+\sqrt{\left(\frac{F}{2 E I}\right)^{2}+\left(\frac{\rho A}{E I}\right) \omega^{2}}}=\sqrt{-\frac{\bar{k}^{2}}{2}+\sqrt{\frac{\bar{k}^{4}}{4}+\bar{\beta}^{4}}} \\
& \alpha_{2}=l \sqrt{\frac{F}{2 E I}+\sqrt{\left(\frac{F}{2 E I}\right)^{2}+\left(\frac{\rho A}{E I}\right) \omega^{2}}}=\sqrt{\frac{\bar{k}^{2}}{2}+\sqrt{\frac{\bar{k}^{4}}{4}+\bar{\beta}^{4}}}
\end{aligned}
$$

where $\bar{k}^{2}=F l^{2} / E I$ and $\bar{\beta}^{2}=\omega l^{2} \sqrt{\rho A / E I}$ are dimensionless factors.

The spring of EVEH loaded with heavy proof mass in this work can be modeled as a clamped-free beam with tensile axial load. The boundary conditions of the spring are given as follows:

$$
\begin{gathered}
\text { Clamped end: } \bar{Y}(0)=0 \quad \bar{Y}^{\prime}(0)=0 \\
\text { Free end: } \bar{Y}^{\prime \prime}(l)=0 \quad \bar{Y}^{\prime \prime \prime}(l)+\bar{k}^{2} \bar{Y}^{\prime}(l)=0
\end{gathered}
$$

Insert conditions (5) and (6) into Equation (3) yields the characteristic equation below:

$$
2 \bar{\beta}^{4}+\left(2 \bar{\beta}^{4}+\bar{k}^{4}\right) \cosh \alpha_{1} \cos \alpha_{2}-\bar{\beta}^{2} \bar{k}^{2} \sinh \alpha_{1} \sin \alpha_{2}=0
$$

As $F($ or $\bar{k}$ ) increase, the frequencies decrease until $F$ equals the critical or Euler's buckling load $F_{c r}$ of the beam. The value of $F_{c r}$ can be obtained by putting $\bar{\beta}^{2}=0$ in Equation (7) which results in the relationship $\cos \bar{k}_{n}=0$. For the first mode:

$$
F_{c r}=\pi^{2} E I / 4 l^{2}
$$

The Rayleigh expression for the fundamental natural frequency of unloaded uniform beam is:

$$
\omega_{0}^{2}=\int_{0}^{l} E I\left(\frac{\mathrm{d}^{2} y}{\mathrm{~d} x^{2}}\right)^{2} \mathrm{~d} x / \int_{0}^{l} \rho A y^{2} \mathrm{~d} x
$$

The fundamental natural frequency of tensile beam is:

$$
\omega^{2}=\left(\int_{0}^{l} E I\left(\frac{\mathrm{d}^{2} y}{\mathrm{~d} x^{2}}\right)^{2} \mathrm{~d} x+F \int_{0}^{l}\left(\frac{\mathrm{d} y}{\mathrm{~d} x}\right)^{2} \mathrm{~d} x\right) / \int_{0}^{l} \rho A y^{2} \mathrm{~d} x
$$

The critical or Euler's buckling load $F_{c r}$ for the same beam is:

$$
\int_{0}^{l} E I\left(\frac{\mathrm{d}^{2} y}{\mathrm{~d} x^{2}}\right)^{2} \mathrm{~d} x+F \int_{0}^{l}\left(\frac{\mathrm{d} y}{\mathrm{~d} x}\right)^{2} \mathrm{~d} x=0
$$

Substituting Equations (9) and (11) into Equation (10) gives:

$$
f=f_{0} \sqrt{1+\frac{F}{F_{c r}}}
$$

where $f_{0}$ is the natural frequency of uncompressed beam, $f$ is the fundamental natural frequency of tensile beam, $F_{c r}$ is the critical or Euler's buckling load for beam, and $F$ is the applied axial tensile load.

As the magnet asserts a large axial force to the spring, we assume the spring to be a clamped-free beam with uniform cross-section and constant axial tensile load. The factor that has been omitted in the model is the dynamic deformation of the spring during 
oscillation and the combined torsional movement of the spring. Therefore, the fundamental resonant frequency can be approximated is given by:

$$
f=\frac{1}{2 \pi} \sqrt{\frac{k_{e f f}\left(F_{c r}+F\right)}{(m+M) F_{c r}}}
$$

where $k_{e f f}$ is the effective stiffness of the spring calculated by the finite-element method (FEM), $m$ is the total mass of the beam, and $M$ is the mass of the disc magnet, the applied axial tensile load $F$ is equal to the gravity of disc magnet. Equation (13) is a rough estimation as it assumes a spring with uniform cross-section. In order to verify the fundamental frequency calculated by (13), FEM simulation based on the computed value of the spring is conducted by using COMSOL Multiphysics. The fundamental natural frequency of the EVEH analytically calculated by (13) and simulated by FEM shows good agreement with each other, as shown in Figure 2. The resonant frequency of the spring decreases when the mass of the magnet increases, the decreasing rate is higher when the mass is smaller.

Table 1. Critical parameters used the resonant frequency calculation.

\begin{tabular}{cc}
\hline Parameters & Values \\
\hline$k_{\text {eff }}(\mathrm{N} \cdot \mathrm{m})$ & 25.2 \\
$P_{c r}(\mathrm{~N})$ & 2.514 \\
$m(\mathrm{~g})$ & 0.0754 \\
\hline
\end{tabular}

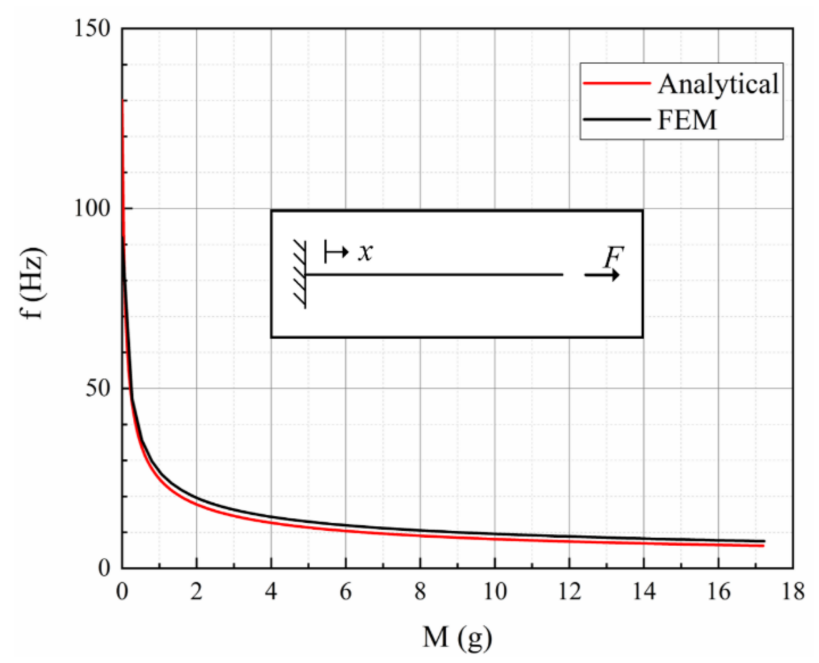

Figure 2. The fundamental resonant frequency versus the mass of the magnet. The computation is based on the values given in Table 1.

\subsection{Modal Analysis}

Due to potential collision between the magnet and the coil, it is difficult to model the behavior of the EVEH device. In order to study the dynamic characteristics of the spring, modal analysis on the resonant mode and frequency of the proposed EVEH is conducted via FEM by using COMSOL Multiphysics, as shown in Figure 3. The model included the gravity of the magnet. Moreover, the primary resonant mode is the bending mode of the spring, similar to the bending of a cantilever. The simulated resonant frequency of the bending mode is $9.4 \mathrm{~Hz}$. Nevertheless, the FEM simulated frequency of the bending mode is not accurate, as the bending mode is strongly influenced by the axial stress in the spring. It is difficult to include the stress in the FEM model since the stress distribution within the spring is rather complex. The main sources of the stress in the spring include mean residual stress and stress gradient from the manufacturing process. The second and the third resonant modes are torsional mode and in-plane sliding mode at $21.9 \mathrm{~Hz}$ and $27.7 \mathrm{~Hz}$, 
respectively. The factors ignored in the FEM mode is the imperfect clamping condition at the spring as well as inaccuracy in the geometry of the device from the assembly process.

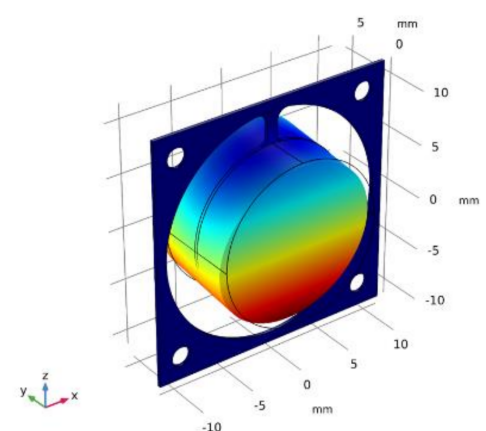

(a) Bending $(9.4 \mathrm{~Hz})$

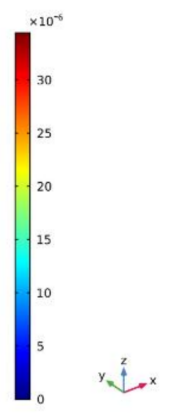

)
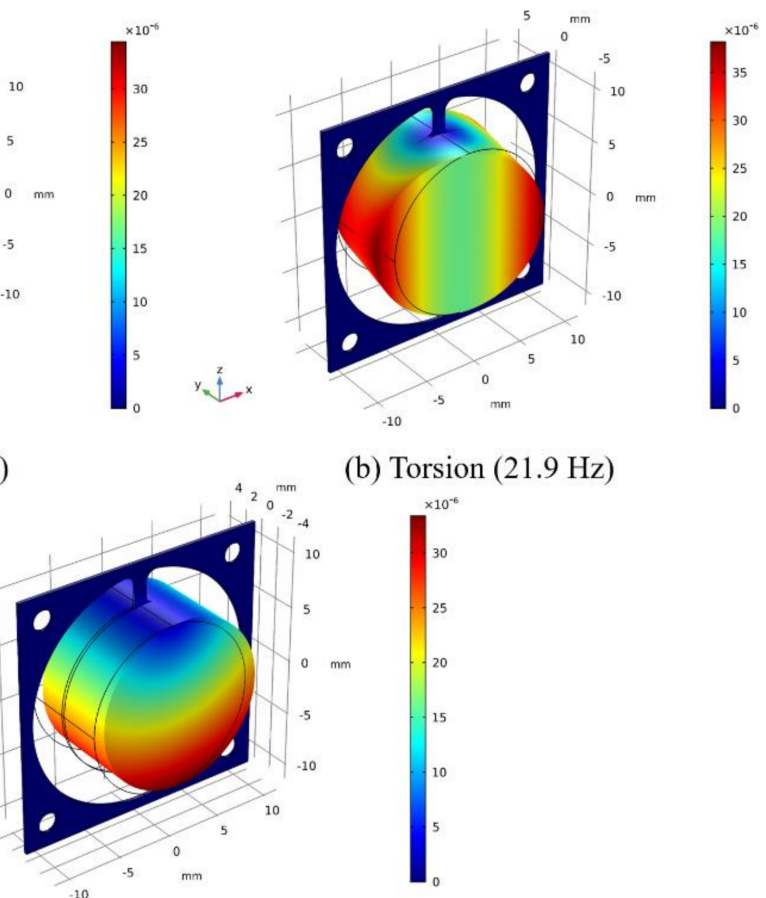

(b) Torsion $(21.9 \mathrm{~Hz})$

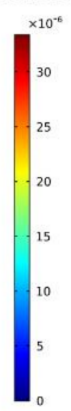

(c) Sliding $(27.7 \mathrm{~Hz})$

Figure 3. FEM modal analysis of the proposed EVEH.

\section{Experimental Procedures}

\subsection{Fabrication and Assembly of the Device}

The proposed EVEH is successfully fabricated and assembled, as shown in Figure 4. The measured key geometrical parameters of the EVEH are listed in Table 2. Figure 4a shows the assembled EVEH device. Figure $4 \mathrm{~b}$ shows the side view of the assembled device. The two magnets are suspended between two sets of polymer coil stacks. The magnet layer and coil stacks are assembled by using long bolts and spacers. The distance between the magnets and coils can be adjusted by tuning the thickness of the spacers. Figure $4 \mathrm{c}, \mathrm{d}$ shows the disassembled and assembled polymer coil stack. In the photos, only 5 coil layers are used for the purpose of simplicity. Spiral copper coils with thickness of $12 \mu \mathrm{m}$ are electroplated and etched on a $180 \mu \mathrm{m}$-thick polyimide substrate to realize the polymer coil layer. Each of the coil layers has two planar coils electroplated on both sides of the polyimide substrates with opposite winding directions, connected by the through-polyimide vias in the center of the coil. Both planar copper coils are covered by thin insulation layer to avoid short circuit when bring into contact. The large electrode pads at the edge of the coil layers connect different layers in series when being stacked. Dummy electrodes with no electrical connection are also electroplated and patterned on the opposite side of the actual electrodes, providing mechanical symmetry and stability in the multi-layer stack. In the EVEH device used for the studies in the next sections, each of the coil stacks consists of 14 coil layers, sandwiched and clamped between two rigid FR4 frames. The FR4 frames are manufactured with standard PCB technologies, with pads for wiring and bolt holes for alignment and clamping. The pads on the FR4 layer are through holes with Sn electroplated on the inner surface and on the back, in order to interface the electrodes on polymer coil and external wiring. Two N52 NdFeB magnets with thicknesses of $4 \mathrm{~mm}$ and diameter of $15 \mathrm{~mm}$ are fixed together by magnetic force with a polyimide circular plate sandwiched in between. The magnets are suspended by a single string polyimide spring connected to the polyimide circular plate. The polyimide 
springs and circular plates are realized by laser cutting an entire piece of polyimide foil. The polyimide spring is anchored to a square polyimide frame clamped between two rigid FR4 frames during assembly, as shown in Figure 4e.
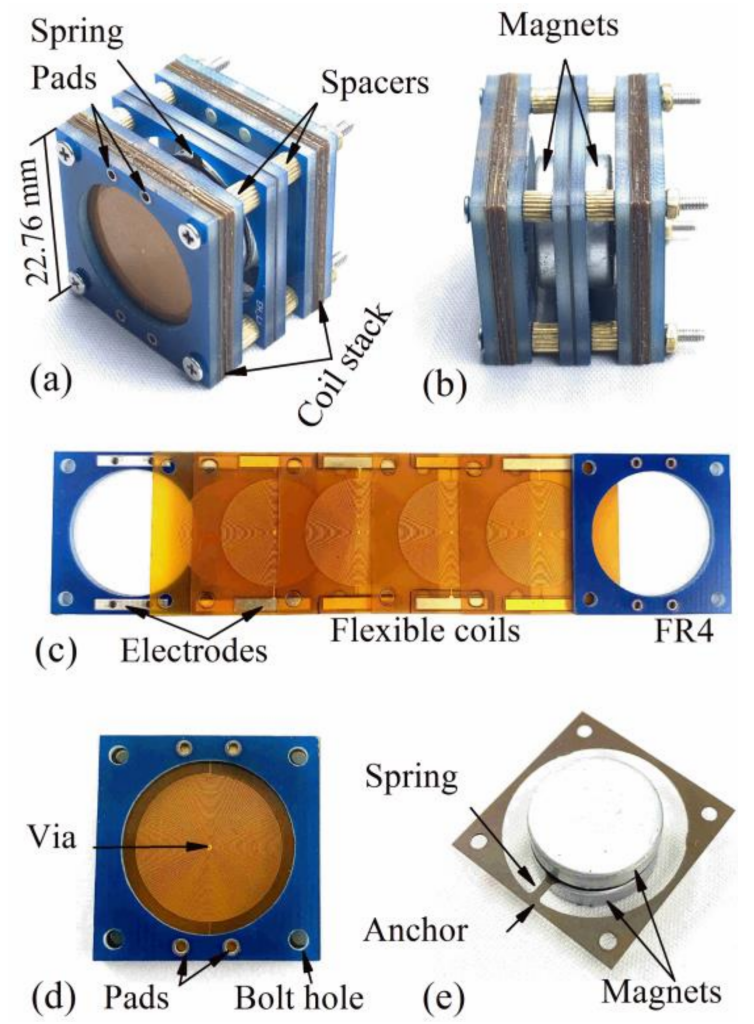

Figure 4. Photos of the (a) fabricated EVEH device, (b) side view of the EVEH device, (c) flexible polymer coils and FR4 boards, (d) assembled flexible coil stack, and (e) magnets and spring.

Table 2. Measured geometrical parameters of the fabricated EVEH.

\begin{tabular}{cc}
\hline Parameters & Measured Values \\
\hline Size of the device $(\mathrm{L} \times \mathrm{W} \times \mathrm{H})(\mathrm{mm})$ & $22.8 \times 22.8 \times 15.6$ \\
Coil thickness $(\mathrm{mm})$ & 0.012 \\
Coil line width $(\mathrm{mm})$ & 0.05 \\
Spacing between coil lines $(\mathrm{mm})$ & 0.05 \\
Number of coil turns per layer & 98 \\
Length of the spring $(\mathrm{mm})$ & 2.8 \\
Width of the spring $(\mathrm{mm})$ & 1.0 \\
Thickness of the spring $(\mathrm{mm})$ & 0.3 \\
Diameter of the magnet $(\mathrm{mm})$ & 15.00 \\
Thickness of the magnet $(\mathrm{mm})$ & 4 \\
Magnet-coil gap $(\mathrm{mm})$ & 2.61 \\
\hline
\end{tabular}

\subsection{Characterization Setup}

The fabricated EVEH device is characterized using a setup shown in Figure 5a. The EVEH is mounted on a piezo shaker, which is actuated by a sinusoidal signal from a signal generator and amplified by a power amplifier. An accelerometer is mounted between the EVEH and the shaker to measure the applied acceleration. A NI USB-6211 data acquisition board is used to acquire the output signal of the EVEH, signal generator, power amplifier, and accelerometer with a sampling rate of 10,000 samples per second. A 4th-order Butterworth low-pass filter with a cut-off frequency of $1000 \mathrm{~Hz}$ is implemented in the LabVIEW software to remove the high-frequency noise from the signals. When measuring the $\mathrm{Z}$ direction operation of the $\mathrm{EVEH}$, the device is mounted in the way that 
the surface of the disc magnet is perpendicular to the direction of the vibration, as shown in Figure $5 \mathrm{~b}$. When measuring the $\mathrm{X}$ direction operation of the EVEH, the EVEH is 90 degree rotated so that the surface of the disc magnet aligns with the direction of the vibration, as shown in Figure 5c.

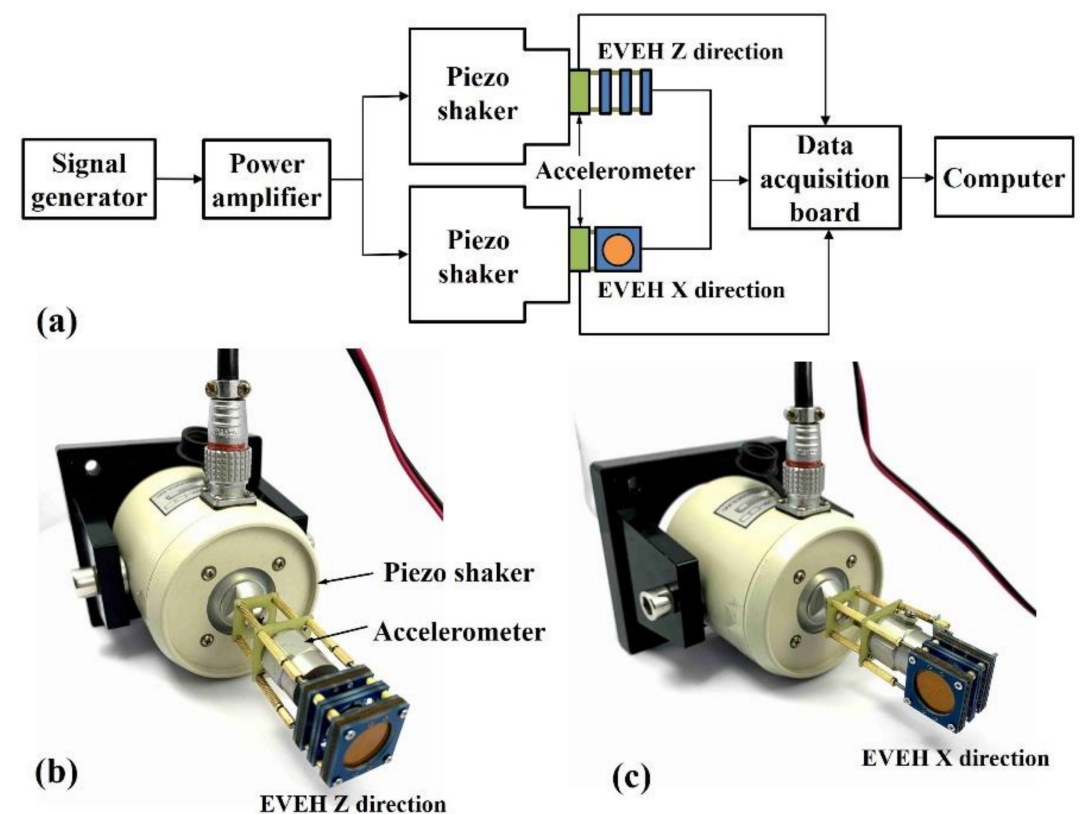

Figure 5. (a) Schematic illustration of the setup for characterizing the EVEH device; (b) photo of the $\mathrm{Z}$ direction measurement setup; and (c) photo of the $\mathrm{X}$ direction measurement setup.

\section{Results and Discussion}

\subsection{Open-Circuit Characteristics}

The open-circuit output voltage of the EVEH under sinusoidal excitations with amplitude of $\pm 1 \mathrm{~g}$ and different frequency is measured as a function of time, as shown in Figure 6. Both the output voltages on $\mathrm{Z}$ and $\mathrm{X}$ directions show sinusoidal characteristics but with distorted waveforms, i.e., oscillation at the peaks of Z-direction output and varying amplitude of the $\mathrm{X}$-direction output. The oscillation on $\mathrm{Z}$ direction is caused by collision between the magnet and the coil. The varying amplitude of the X-direction output is induced by the slight collision between the magnet and FR4 frame. In the next sections, the output voltage values of the EVEH are average values of 10 periods, in order to reduce the inaccuracy from the influence of the oscillations.

The output voltage is small when the frequency of the excitation vibration is far from the resonant frequency of the EVEH. When the vibration frequency gets closer to the resonant frequency of the EVEH, both the output voltage and distortion increase significantly on both $Z$ and $X$ directions, as a result of the increased movement of the magnet. The output voltages of the top and bottom coils are almost identical for the $X$-direction vibration. In comparison, the Z-direction output voltages of the top and bottom coils have similar positive peaks but slightly different negative peaks, possibly as a result of slightly different movement of the magnet with respect to the top and bottom coils due to collision. At $15 \mathrm{~Hz}$, the maximum output voltages are $0.90 \mathrm{~V}$ (top coil) and $0.95 \mathrm{~V}$ (bottom coil) on $\mathrm{Z}$ direction, $0.28 \mathrm{~V}$ (top coil) and $0.31 \mathrm{~V}$ (bottom coil) on $\mathrm{X}$ direction, respectively. The maximum output voltage on $Z$ direction is larger than on $X$ direction, because the thin and wide spring is easier to be bended (in $Z$ direction) than to be twisted or bended (in $\mathrm{X}$ direction). The low-stiffness yet robust polyimide spring enables large and multi degree-of-freedom movement of the magnet while maintains the reliability of the system. Commonly used rigid springs based on silicon and FR4 may not be able to withstand the large movement amplitude of the magnet and mechanical shock induced by the collision between the magnet and coil. 

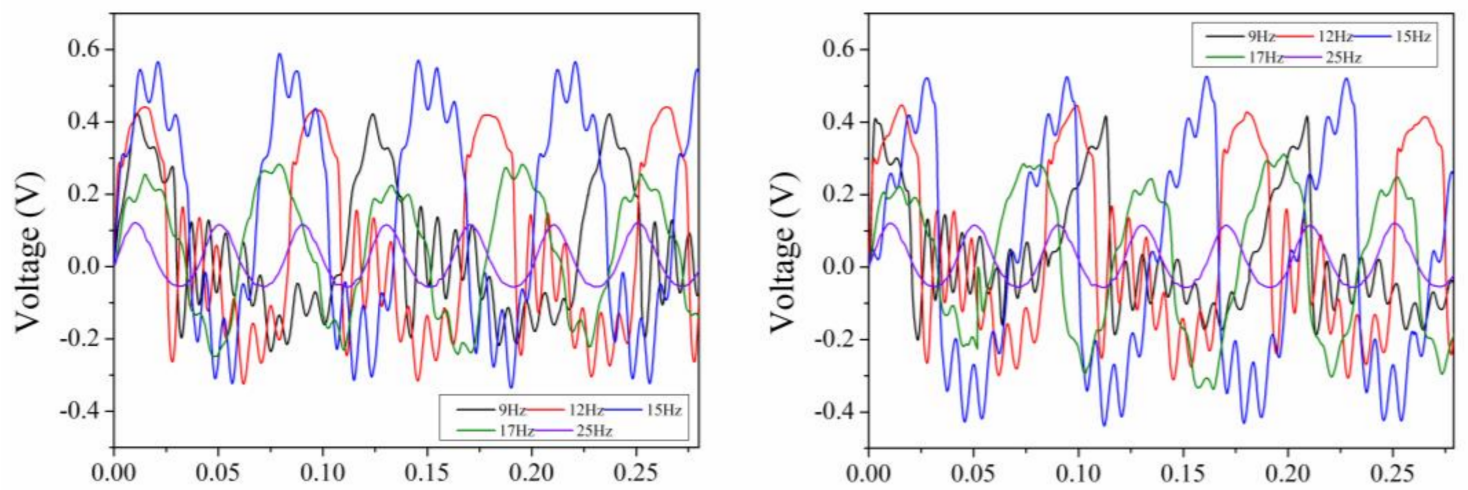

(a)

Time (s)
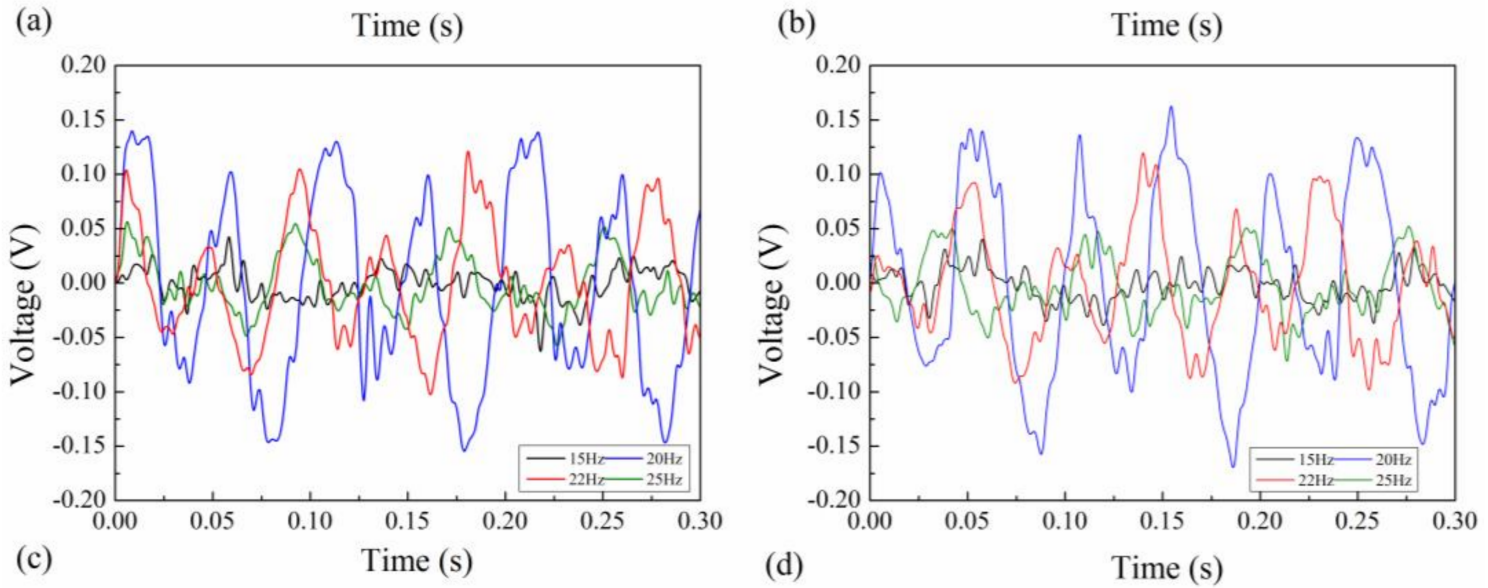

Figure 6. The time-dependent open-circuit output voltage of the EVEH on (a) Z-direction in top coil; (b) Z-direction in bottom coil; (c) X-direction in top coil; and (d) X-direction in bottom coil.

The peak-to-peak open-circuit output voltage of the EVEH as a function of frequency is shown in Figure 7. The output voltages of the EVEH on $\mathrm{Z}$ and $\mathrm{X}$ directions are measured by both sweeping up and sweeping down the frequency. The output voltage in both top and bottom coils are measured.

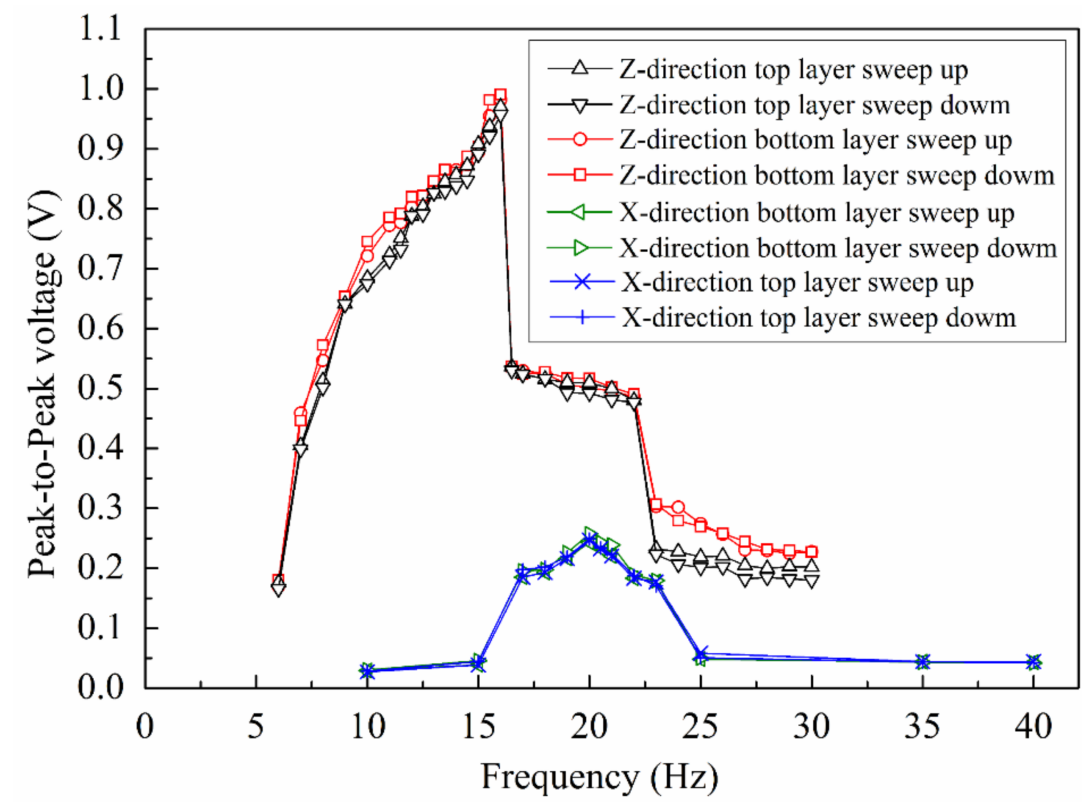

Figure 7. The frequency-dependent open-circuit output voltage of the EVEH in both top and bottom coils, on both $\mathrm{Z}$ and $\mathrm{X}$ directions. 
On $\mathrm{Z}$ direction, two resonance peaks have been observed at $16 \mathrm{~Hz}$ and $20 \mathrm{~Hz}$, respectively. The corresponding resonant modes are bending $(16 \mathrm{~Hz})$ and torsional $(20 \mathrm{~Hz})$ modes as predicted by the FEM simulation. The resonant frequency of the torsional mode shows good agreement with the FEM simulation $(21.9 \mathrm{~Hz})$. However, there is discrepancy between the measured and simulated resonant frequency of the bending mode $(16 \mathrm{~Hz}$ versus $9.4 \mathrm{~Hz}$ ), possibly due to the dynamic axial stress in the spring which is not accurately modeled in the FEM. In addition, we have observed the movement of the magnet and discovered that none of the resonant modes of the magnet is a simple motion, as a result of multiple degree of freedom of the spring and the magnet-coil collision. For instance, the bending mode is rather a bending movement combined with torsional movement, and the torsional mode is rather a torsional movement combined with an in-plane sliding motion.

The maximum output voltage of the EVEH is $0.99 \mathrm{~V}$ at $16 \mathrm{~Hz}$ and $0.52 \mathrm{~V}$ at $20 \mathrm{~Hz}$. The difference in the measured maximum outputs in the top and bottom coils is within $0.03 \mathrm{~V}$. There is an abrupt decrease in the output voltage once the frequency is higher than the resonant frequency of the EVEH device. The abrupt decrease often originates from the nonlinear behavior of spring as a result of the axial stress or the overlap between two resonant modes. However, no hysteresis has been observed between the sweepup and sweep-down (in frequency) characteristics of the EVEH, indicating the absence of nonlinearity in the spring. Therefore, the abrupt decrease may originate from the overlapping between the two resonant modes, which actually effectively expanded the bandwidth of the device. The peak of the torsional mode on $\mathrm{Z}$ direction is not obvious, possibly as a result of the overlap between the peak of the torsional mode and the abrupt decrease in amplitude of the bending mode. It is worth noting that the EVEH outputs around $0.2 \mathrm{~V}$ of voltage even at the non-resonant frequencies, as a result of the low-stiffness pendulum-like suspension of the magnet. On $X$ direction, only the torsional mode of the EVEH is excited and observed. A maximum output voltage of $0.26 \mathrm{~V}$ is observed at the resonant frequency of $20 \mathrm{~Hz}$. The difference in the measured maximum outputs in the top and bottom coils is within $0.01 \mathrm{~V}$. Vibrations on specific directions can only excite specific corresponding resonant modes, e.g., vibration on $\mathrm{Z}$ direction is capable of exciting both the bending and torsional mode of the magnet, whereas vibration on $X$ direction is only capable of exciting torsional mode of the magnet. This is determined by the design of the spring: a thin and wide spring is easy to be bended towards the vertical direction rather than the parallel direction of its wide surface. This trend is more obvious when the ratio between the width and thickness is larger.

\subsection{Impedance Matching}

In practical applications, an EVEH device is often used as a power supply and connected to an electric load. Therefore, it is important to study the output characteristics of the EVEH under different load conditions. In this section, the output voltage and power of the EVEH device is measured while its output is connected to load resistors with various values.

The output peak-to-peak closed-circuit voltage and power of the EVEH device on both $\mathrm{Z}$ and $\mathrm{X}$ directions is plotted versus the load resistance, as shown in Figure 8. The data in the figure are obtained with an applied excitation vibration of $\pm 1 \mathrm{~g}$ and frequencies of $16 \mathrm{~Hz}$ ( $\mathrm{Z}$ direction) and $20 \mathrm{~Hz}$ (X direction). On $\mathrm{Z}$ direction, the maximum output power of the EVEH is $33.7 \mu \mathrm{W}$ with an optimal load resistance of $940 \Omega$. On $X$ direction, the maximum output power of the EVEH is $3.2 \mu \mathrm{W}$ with an optimal load resistance of $940 \Omega$. The power on $\mathrm{X}$ direction is 10.5 times smaller than on $\mathrm{Z}$ direction, because the power is proportional to the square of the voltage. The optimal load value is similar as the measured coil resistance of $937 \Omega$. The slight difference between the optimal load and the coils resistance originates from the measurement inaccuracy as well as the ignored resistance of the wires. 

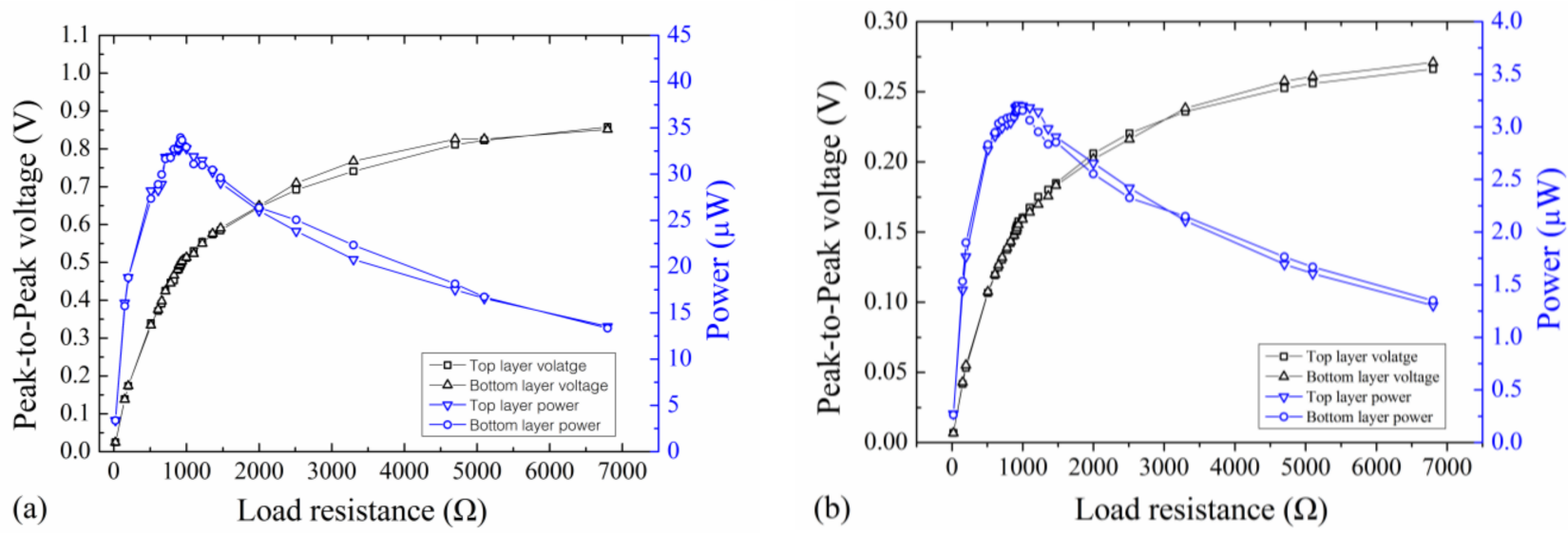

Figure 8. The closed-circuit output voltage and power of the EVEH as a function of load resistance on (a) Z direction and (b) X direction.

\subsection{Closed-Circuit Characteristics}

The closed-circuit output voltage and power of the EVEH as a function of frequency is shown in Figure 9. Figure 9a shows the output voltage and power on $\mathrm{Z}$ direction under an applied vibration excitation of $\pm 1 \mathrm{~g}$. Two resonance modes of bending and torsion are visible in the figure. At the dominant resonant frequency of $16 \mathrm{~Hz}$, a maximum output voltage of $0.51 \mathrm{~V}$ and a power of $35.1 \mu \mathrm{W}$ are measured in the top coil. Correspondingly, a maximum output voltage of $0.50 \mathrm{~V}$ and a power of $33.6 \mu \mathrm{W}$ are measured in the bottom coil at $16 \mathrm{~Hz}$. Combining output of the top and bottom coils, the device is capable of generating a power of $68.7 \mu \mathrm{W}$ in total. No hysteresis has been observed in the frequency sweep-up and sweep-down measurements. The maximum output power of $68.7 \mu \mathrm{W}$ is 429 times higher than the maximum output power of $160 \mathrm{nW}$ at $1 \mathrm{~g}$ vibration of the EVEH with similar technology reported in 2016 [8] and 159 times higher than the maximum output power of $0.43 \mu \mathrm{W}$ of the EVEH with similar technology and size reported in 2017 [6]. The output power is lower than the work based on stacked coils reported in 2018 [7], due to the increased magnet-coil distance. Nevertheless, the EVEH presented in this work is capable of harvesting vibrations on two different directions, which has not been realized in previous works. With a volume of $8.1 \mathrm{~cm}^{3}$, the proposed device has a normalized power density (NPD) of $8.48 \mu \mathrm{W} / \mathrm{cm}^{3} \cdot \mathrm{g}^{2}$, which is higher than several similar polymerbased energy harvesters, e.g., 42 times higher than the NPD of $0.2 \mu \mathrm{W} / \mathrm{cm}^{3} \cdot \mathrm{g}^{2}$ of an electromagnetic/triboelectric hybrid polymer energy harvester [20], 1696 times higher than the NPD of $0.005 \mu \mathrm{W} / \mathrm{cm}^{3} \cdot \mathrm{g}^{2}$ of an electromagnetic energy harvester based on flexible coil and liquid spring [21]. In [22], the NPD of an electromagnetic energy harvester based on magnetic spring $\left(1.97 \mathrm{~mW} / \mathrm{cm}^{3} \cdot \mathrm{g}^{2}\right)$ is 232 times higher than this work, because it works at very low acceleration. The magnetic spring of the device enables operation at very low acceleration $(0.4 \mathrm{~g})$ but increases the volume of the device $\left(194.5 \mathrm{~cm}^{3}, 24\right.$ times larger than the proposed EVEH device). Nevertheless, there are other advantages of the magnetic spring technology, e.g., extended lifetime and reduced negative stiffness spring [23]. The combination of non-linear resonance and multi-mode operation of the EVEH on Z direction provide a higher bandwidth than traditional EVEH devices with single resonance mode. Figure $9 \mathrm{~b}$ shows the output voltage and power on $\mathrm{X}$ direction under an applied vibration excitation of $\pm 1.5 \mathrm{~g}$. On $X$ direction, the dominant mode is the torsional mode at $20 \mathrm{~Hz}$, where a maximum output voltage of $0.15 \mathrm{~V}$ and power of $2.96 \mu \mathrm{W}$ are measured in the top coil and a maximum output voltage of $0.14 \mathrm{~V}$ and power of $2.56 \mu \mathrm{W}$ are measured in the bottom coil. The bending mode has a very small amplitude, evidenced by the slight change in the slope of the curve around $16 \mathrm{~Hz}$. The resonant frequency of the proposed device is among the lowest of the reported electromagnetic energy harvesters. If further reduction in the operation frequency is needed, one may consider the use of non-resonant energy harvesters such as the triboelectric-based devices [23,24]. 

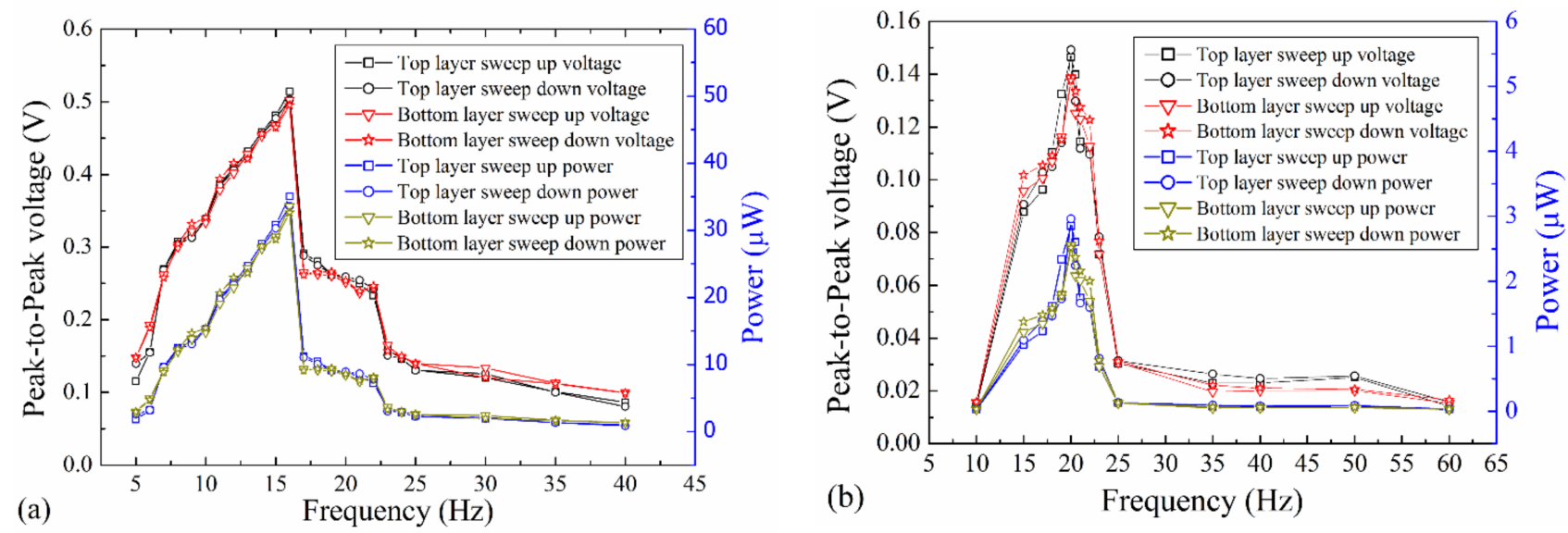

Figure 9. The closed-circuit output voltage of the EVEH as a function of frequency on (a) Z direction and (b) X direction.

\subsection{Acceleration Test}

The output of the EVEH under different vibration levels is studied on both $\mathrm{Z}$ and $\mathrm{X}$ directions. On $\mathrm{Z}$ direction, the open-circuit output voltage of the EVEH is measured under excitation of $\pm 0.2- \pm 1 \mathrm{~g}$, as shown in Figure 10a. The output voltage increases as the vibration amplitude increases. The voltage increases first rapidly between $\pm 0.2 \mathrm{~g}$ and $\pm 0.4 \mathrm{~g}$ and then gradually between $\pm 0.4 \mathrm{~g}$ and $\pm 1 \mathrm{~g}$. The saturation in the increase of output voltage is due to the limited motion of the magnet that can be achieved within the small coil-magnet gap. The output voltage increases from $0.18 \mathrm{~V}$ at $\pm 0.2 \mathrm{~g}$ to $0.98 \mathrm{~V}$ at $\pm 1 \mathrm{~g}$. On $\mathrm{X}$ direction, the open-circuit output voltage of the EVEH is measured under excitation of $\pm 0.4- \pm 3 \mathrm{~g}$, as shown in Figure 10b. The output voltage increases rapidly in the range of $\pm 0.4- \pm 1 \mathrm{~g}$, and saturates gradually after $\pm 1 \mathrm{~g}$. The output voltage increases from $0.02 \mathrm{~V}$ at $\pm 0.4 \mathrm{~g}$ to $0.41 \mathrm{~V}$ at $\pm 3 \mathrm{~g}$. The dependence of the output on acceleration suggests that the EVEH is suitable to be used at low acceleration level on $\mathrm{Z}$ direction and at high acceleration on $X$ direction. Using the EVEH at high acceleration level may induce intensive collision between the magnet and the coil, which will deteriorate the reliability of the device.
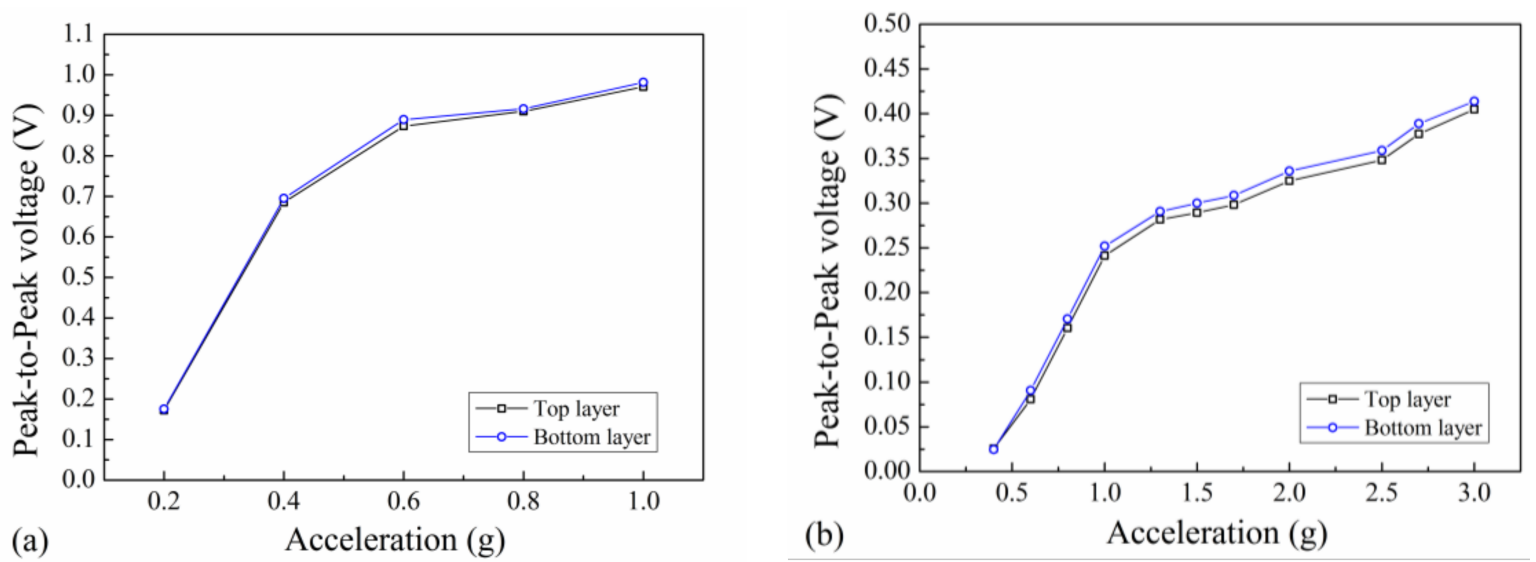

Figure 10. The open-circuit output voltage of the EVEH as a function of acceleration level on (a) Z-direction and (b) X-direction.

\section{Conclusions}

In this work, a low frequency electromagnetic vibrational energy harvester (EVEH) with two degrees of freedom and two resonant modes is designed, fabricated, and characterized. The proposed EVEH is implemented by suspending a disc magnet in a pendulum fashion by a polymer spring between two sets of polymer coil stacks. The fabricated EVEH is capable of harvesting vibration energy on two directions with an extended bandwidth. With a sinusoidal acceleration of $\pm 1 \mathrm{~g}$ in amplitude on $\mathrm{Z}$ direction, a peak-to-peak closed- 
circuit output voltage of $0.51 \mathrm{~V}$ (open-circuit voltage: $1 \mathrm{~V}$ ) and an output power of $35.1 \mu \mathrm{W}$ are measured, at the resonant frequency of $16 \mathrm{~Hz}$. The two coils in the device output a power of $68.7 \mu \mathrm{W}$ in total. With a sinusoidal acceleration of $\pm 1.5 \mathrm{~g}$ in amplitude on $\mathrm{X}$ direction, a peak-to-peak output voltage of $0.14 \mathrm{~V}$ and power of $2.56 \mu \mathrm{W}$ are measured, at the resonant frequency of $20 \mathrm{~Hz}$. In future work, the size and structure of the proposed EVEH can be still optimized from the aspect of design and fabrication process. In addition, despite the excellent energy harvesting capabilities, the proposed EVEH does have issues such as magnet-coil collision, which might reduce the reliability and lifetime of the EVEH device. These issues must be considered in the future design of the EVEH devices.

Author Contributions: Conceptualization, Y.L., K.T. and X.W.; methodology, C.Z. and D.Q.; investigation, S.Z. and X.W.; data curation, K.T.; writing-original draft preparation, Y.L.; writing-review and editing, Y.L. and K.T.; visualization, C.Z.; supervision, Y.L. All authors have read and agreed to the published version of the manuscript.

Funding: This research was funded by the National Key Research and Development Program of China (2018YFF01010902) and the National Natural Science Foundation of China (51705405).

Acknowledgments: The authors would like to acknowledge Qi Cao and Jiaxing Li for fruitful discussions in the EVEH characterization.

Conflicts of Interest: The authors declare no conflict of interest.

\section{References}

1. Priya, S.; Inman, D.J. Energy Harvesting Technologies; Springer: Berlin/Heidelberg, Germany, 2009.

2. Mallick, D.; Amann, A.; Roy, S. High figure of merit nonlinear microelectromagnetic energy harvesters for wideband applications. J. Microelectromech. Syst. 2016, 26, 273-282. [CrossRef]

3. Podder, P.; Constantinou, P.; Mallick, D.; Amann, A.; Roy, S. Magnetic tuning of nonlinear MEMS electromagnetic vibration energy harvester. J. Microelectromech. Syst. 2017, 26, 539-549. [CrossRef]

4. Tao, K.; Wu, J.; Tang, L.; Xia, X.; Lye, S.W.; Miao, J.; Hu, X. A novel two-degree-of-freedom MEMS electromagnetic vibration energy harvester. J. Micromech. Microeng. 2016, 26, 035020. [CrossRef]

5. Tan, Y.; Dong, Y.; Wang, X. Review of MEMS electromagnetic vibration energy harvester. J. Microelectromech. Syst. 2016, 26, 1-16. [CrossRef]

6. Li, Y.; Zhang, W.; Zhang, Y.; Cao, J. A batch-fabricated electromagnetic energy harvester based on flex-rigid structures. Sens. Actuators A Phys. 2017, 263, 571-579. [CrossRef]

7. Li, Y.; Cao, Q.; Zhang, W.; Zhang, Y.; Cao, J.A. A miniaturized electromagnetic energy harvester with volt-level output based on stacked flexible coils. Smart Mater. Struct. 2018, 27, 115040. [CrossRef]

8. Chiu, Y.; Hong, H.-C.; Hsu, W.-H. Wideband vibrational electromagnetic energy harvesters with nonlinear polyimide springs based on rigid-flex printed circuit boards technology. Smart Mater. Struct. 2016, 25, 125014. [CrossRef]

9. Roundy, S.; Takahashi, E. A planar electromagnetic energy harvesting transducer using a multi-pole magnetic plate. Sens. Actuators A Phys. 2013, 195, 98104. [CrossRef]

10. Han, M.; Yuan, Q.; Sun, X.; Zhang, H. Design and fabrication of integrated magnetic MEMS energy harvester for low frequency applications. J. Microelectromech. Syst. 2013, 23, 204212. [CrossRef]

11. Liu, H.; Qian, Y.; Wang, N.; Lee, C. An in-plane approximated nonlinear MEMS electromagnetic energy harvester. J. Microelectromech. Syst. 2013, 23, 740-749. [CrossRef]

12. Liu, H.; Qian, Y.; Lee, C. A multi-frequency vibration-based MEMS electromagnetic energy harvesting device. Sens. Actuators A Phys. 2013, 204, 37-43. [CrossRef]

13. Bang, D.H.; Park, J.Y. Micro-fabricated silicon spiral spring based electromagnetic energy harvester. J. Korean Phys. Soc. 2013, 62, 1720-1725. [CrossRef]

14. Han, M.; Li, Z.; Sun, X.; Zhang, H. Analysis of an in-plane electromagnetic energy harvester with integrated magnet array. Sens. Actuators A Phys. 2014, 219, 38-46. [CrossRef]

15. Beeby, S.P.; Torah, R.N.; Tudor, M.J.; Glynne-Jones, P.; O'donnell, T.; Saha, C.R.; Roy, S. A micro electromagnetic generator for vibration energy harvesting. J. Micromech. Microeng. 2007, 17, 1257. [CrossRef]

16. Xu, J.; Tang, J. Multi-directional energy harvesting by piezoelectric cantilever-pendulum with internal resonance. Appl. Phys. Lett. 2015, 107, 213902. [CrossRef]

17. Chen, R.; Ren, L.; Xia, H.; Yuan, X.; Liu, X. Energy harvesting performance of a dandelion-like multi-directional piezoelectric vibration energy harvester. Sens. Actuators A Phys. 2015, 230, 1-8. [CrossRef]

18. Park, J.C.; Park, J.Y. Asymmetric PZT bimorph cantilever for multi-dimensional ambient vibration harvesting. Ceram. Int. 2013, 39, S653-S657. [CrossRef]

19. Bokaian, A. Natural frequency of beam under compressive axial loads. J. Sound Vib. 1988, 126, 49-65. [CrossRef] 
20. Gupta, R.K.; Shi, Q.; Dhakar, L.; Wang, T.; Heng, C.H.; Lee, C. Broadband energy harvester using non-linear polymer spring and electromagnetic/triboelectric hybrid mechanism. Sci. Rep. 2017, 7, 1-13. [CrossRef] [PubMed]

21. Wang, Y.; Zhang, Q.; Zhao, L.; Tang, Y.; Shkel, A.; Kim, E.S. Vibration energy harvester with low resonant frequency based on flexible coil and liquid spring. Appl. Phys. Lett. 2016, 109, 203901. [CrossRef]

22. Aldawood, G.; Nguyen, H.T.; Bardaweel, H. High power density spring-assisted nonlinear electromagnetic vibration energy harvester for low base-accelerations. Appl. Energy 2019, 253, 113546. [CrossRef]

23. Siang, J.; Lim, M.H.; Salman Leong, M. Review of vibration-based energy harvesting technology: Mechanism and architectural approach. Int. J. Energy Res. 2018, 42, 1866-1893. [CrossRef]

24. Tao, K.; Chen, Z.; Yi, H.; Zhang, R.; Shen, Q.; Wu, J.; Tang, L.; Fan, K.; Fu, Y.; Miao, J.; et al. Hierarchical Honeycomb-Structured Electret/Triboelectric Nanogenerator for Biomechanical and Morphing Wing Energy Harvesting. Nano-Micro Lett. 2021, 13, 1-16. [CrossRef] [PubMed] 